\title{
Mathematical modeling of bulk and directional crystallization with the moving phase transition layer
}

\author{
Liubov Toropova ${ }^{1}$, Danil Aseev ${ }^{2}$, Sergei Osipov ${ }^{3}$, and Alexander Ivanov ${ }^{3}$ \\ ${ }^{1}$ Friedrich-Schiller-Universität Jena \\ ${ }^{2}$ UrFU Institute of Natural Sciences and Mathematics \\ ${ }^{3}$ Ural Federal University named after the first President of Russia B N Yeltsin
}

September 24, 2021

\begin{abstract}
This paper is devoted to the mathematical modeling of a combined effect of directional and bulk crystallization in a phase transition layer with allowance for nucleation and evolution of newly born particles. We consider two models with and without fluctuations in crystal growth velocities, which are analytically solved using the saddle-point technique. The particle-size distribution function, solid-phase fraction in a supercooled two-phase layer, its thickness and permeability, solidification velocity, and desupercooling kinetics are defined. This solution enables us to characterize the mushy layer composition. We show that the region adjacent to the liquid phase is almost free of crystals and has a constant temperature gradient. Crystals undergo intense growth leading to fast mushy layer desupercooling in the middle of a two-phase region. The mushy region adjacent to the solid material is filled with the growing solid phase structures and is almost desupercooled.
\end{abstract}

\section{Hosted file}

MMAS_nucl.pdf available at https://authorea.com/users/435243/articles/538397-mathematicalmodeling-of-bulk-and-directional-crystallization-with-the-moving-phase-transition-layer
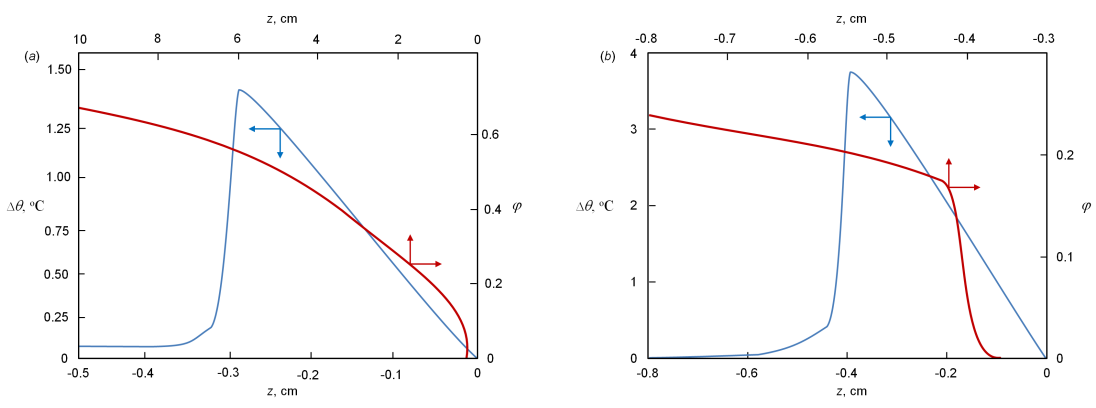

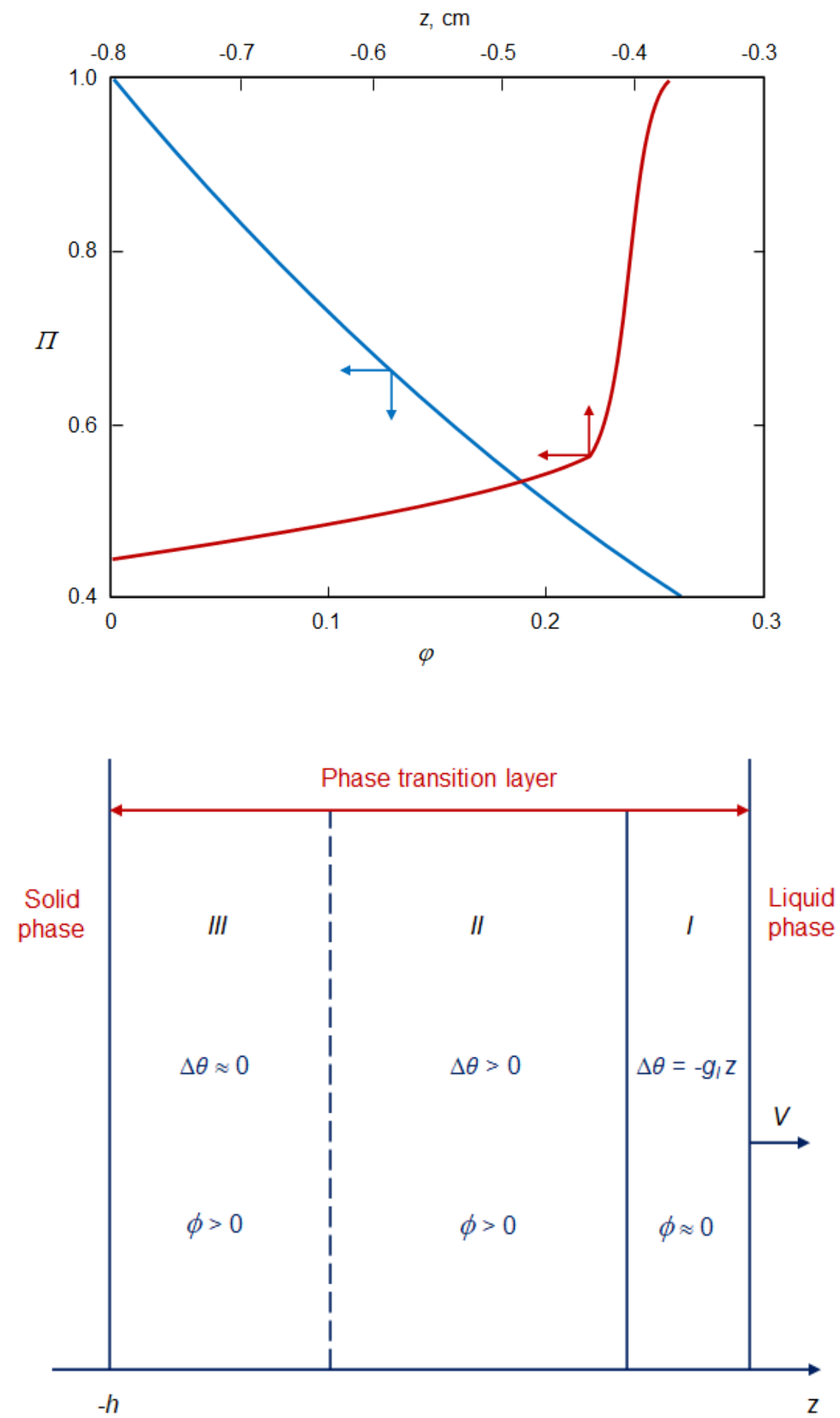